\title{
Injury Risk and Comfort Assessment Applied to Ambulance Transportation
}

\author{
Daniel G. Kyrollos ${ }^{1}$, Terrin Stachiw ${ }^{2}$, James R. Green ${ }^{1}$, Robert G. Langlois ${ }^{2}$ \\ ${ }^{1}$ Systems \& Computer Engineering \\ Carleton University \\ Ottawa, Ontario, Canada \\ daniel.kyrollos@carleton.ca; jrgreen@sce.carleton.ca \\ ${ }^{2}$ Mechanical and Aerospace Engineering \\ Carleton University \\ Ottawa, Ontario, Canada \\ terrin.stachiw@carleton.ca; robert.langlois@carleton.ca
}

\begin{abstract}
Speed humps are an effective means to moderate the speed of traffic but can have adverse effects on the circulation of emergency vehicles. This study aims to use various comfort and injury risk metrics to evaluate the effects of speed hump traversal on the comfort of the passengers. Studies of human comfort during ambulance transport typically consider the peak acceleration and the wholebody vibration dose value from ISO 2631-1. The novel application of other metrics in the ambulance transport scenario, which were derived for naval and aviation settings, provided additional insight to the human biodynamic response and comfort. The following parameters were considered in the study: peak seat acceleration, dynamic response index, peak lumbar acceleration, bandwidth-limited power-spectral density, and the average acceleration onset derivative (average jerk). In-vehicle road tests were conducted in a Type III ambulance on flat-top and sinusoidal speed humps at various traversing speeds. Results from this experiment show that speeds over 20 $\mathrm{km} / \mathrm{hr}$ will result in some degree of discomfort to passengers in the ambulance. Additional experiments are needed to verify the consistency of these parameters, but this study has provided a proof of concept for further studies.
\end{abstract}

Keywords: Speed hump; Ambulance; Injury risk; Comfort

\section{Introduction}

Ride quality is an important consideration in emergency transport and discrete disturbances such as speed humps can adversely impact ride quality. As part of a broader study to investigate ride quality during patient transport, this study considers the impact of speed humps on the comfort and safety of clinical staff accompanying the patient in the back of an ambulance.

Speed humps are traffic calming devices raised above the road surface and are intended to be comfortable for drivers and passengers when traversed below a predetermined design speed, while causing "increasingly more discomfort at higher speeds" [1]. This, however, may not be true for shorter speed humps with a length less than the car's wheelbase, where there exists discomfort when traversing slowly, with less discomfort at high driving speeds [2]. To address this, the shape of speed humps is often standardized to a trapezoidal or semi-circular shape of minimum length and height [1].

The fundamental design objective of speed humps is to cause discomfort to drivers who pass at high speeds to slow the speed of traffic. This objective, however, is troublesome for emergency vehicles as speed humps have been shown to delay the response time when the route includes speed humps [1], [2]. A 2003 survey of paramedics in the UK indicates that paramedics regularly altered their route to avoid speed humps even if it results in an increased response time [3]. Oftentimes, it is impossible to avoid a speed hump, thus motivating this research to determine the maximum traversing speed in consideration of various comfort and injury risk metrics.

\subsection{Review of Human Comfort Parameters}

The acceleration event resulting from an ambulance traversing a speed hump resembles a shock loading with a shortduration (less than 1 second) and high-amplitude acceleration. It is common practice to assess comfort and risk of injury 
under whole-body vibration by applying ISO 2631-1 [4]. The methods of this Standard, however, are intended for longerterm vibration greater than 10 to 20 seconds in duration, where a window of sufficient duration is able to capture the lower frequency range with practical consideration given to the Rayleigh Frequency. When observing passenger comfort during traversal of a speed hump, however, the frequency-weighted root-mean-square acceleration and vibration dose value, as defined in this Standard, are regularly used in previous studies investigating passenger comfort during speed hump traversal [5], [6]. Instead, the methods of ISO 2631-5 [7] are more applicable in the shock loading condition when traversing a speed hump but are intended for long-term exposure to multiple shock events (i.e. regular exposure over several years).

Peterson and Bass [8] and De Alwis [9] provide reviews of various impact injury risk parameters applied to high-speed watercraft for shock loading. Comfort or injury risk under shock loading can be evaluated using the acceleration magnitude and duration, the acceleration onset derivative (the jerk), or by using biodynamic models that are of one or more degrees of freedom (DOFs) [10]. The biodynamic model most commonly applied for evaluating the condition of vertical acceleration of a seated subject is the dynamic response index (DRI) [10]. Li et al. use a 10 DOF vehicle model to perform a multiobjective optimization of ride quality in an ambulance to minimize the peak acceleration of a supine patient's head, chest, and abdomen, as well as that of the driver [11]. The following parameters considered in the study are explained and derived in the subsequent sections: DRI, peak seat acceleration, peak lumbar acceleration (from ISO 2631-5 [7]), bandwidth-limited (BL) power-spectral density (PSD), and the average acceleration onset derivative (average jerk). To the best of the authors' knowledge, the DRI, ISO 2631-5 peak lumbar acceleration, BL PSD, and acceleration fonset derivative have not been applied to assess comfort or injury risk when traversing a speed hump.

\subsubsection{Peak Seat Acceleration}

The peak seat acceleration, $a_{\text {peak }}$, is the maximum value of the acceleration at the seat in the vertical (upward) direction after removing the acceleration due to gravity bias [12]. Various safety and comfort limits of the peak acceleration magnitude and duration are provided by Eiband in Reference [13].

\subsubsection{Dynamic Response Index}

The DRI was developed as a risk of injury model under seated vertical acceleration, such as during aircraft ejection or in armored vehicles in a blast environment. It is a non-dimensional measure of axial spinal compression subject to vertical loads in a seated position for a single shock event. It represents the head and spinal column as an equivalent single degreeof-freedom spring-mass-damper system, as illustrated in Fig. 1, with natural frequency $\omega_{n}=52.9 \mathrm{rad} / \mathrm{s}=8.4 \mathrm{~Hz}$ and damping ratio $\zeta=0.224$, as defined in MIL-DTL-9479E [12]. It can be shown that the transfer function relating the compression of the spinal column, $\Delta(s)$, to an input acceleration at the seat, $A(s)$, is given by

$$
\frac{\Delta(s)}{A(s)}=\frac{1}{s^{2}+23.6992 s+2798.41}
$$

The maximum spinal compression, $\delta_{\text {max }}$, is non-dimensionalized using the natural frequency and acceleration due to gravity, $g$, to give the DRI, as in

$$
\mathrm{DRI}=\frac{\omega_{n}^{2}}{g} \delta_{\max }
$$

This relation can be used to determine the spinal compression time-history, from which $\delta_{\max }$ can be obtained. The Bode magnitude diagram of the transfer function is shown in Error! Reference source not found.. The limiting value of the DRI is given in NATO AEP-55 STANAG 4569 as 17.7, which corresponds to a 10\% risk of serious spinal injury [14].

In conditions of multiple vertical shocks, such as when traversing several consecutive speed humps, the following relation is obtained from Reference [15] to combine the DRI of each event in order to remain below exposure limits for comfort and injury risk

$$
\frac{1}{n_{q}} \sum_{q=1}^{n_{q}} \frac{D R I_{q}}{D R I_{\max }} \leq 1
$$


where $D R I_{\max }$ is the DRI corresponding to the observed number of shocks, $n_{q}$, with magnitude $D R I_{q}$ obtained from the 24 hour exposure limit found in Reference [15].

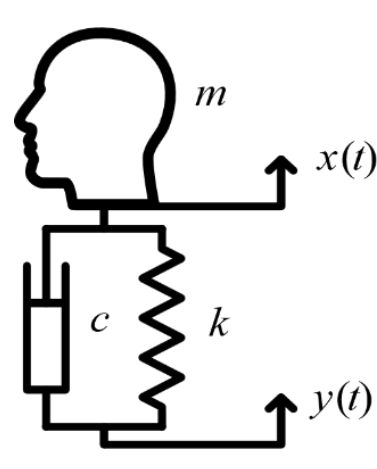

Fig. 1: Schematic of the DRI model

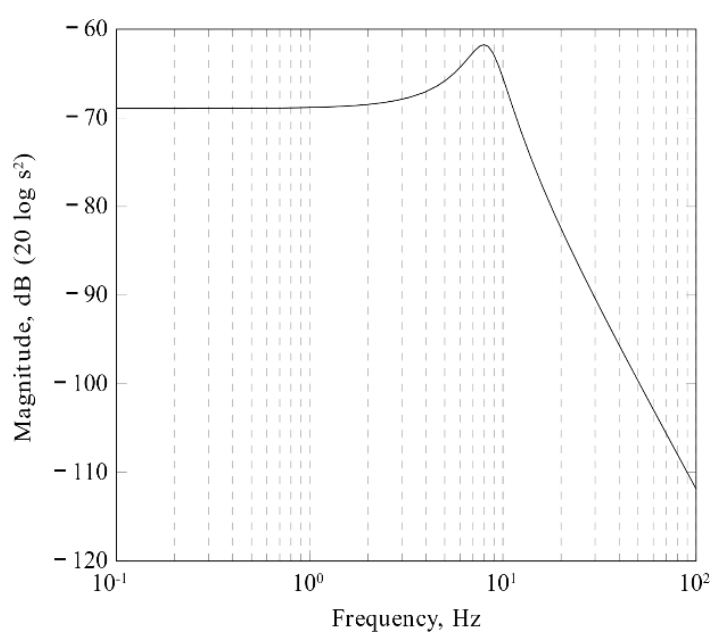

Fig. 2: Bode-magnitude plot of the DRI response

\subsubsection{Peak Lumbar Acceleration}

ISO 2631-5 provides a method to evaluate human injury risk when exposed to repeated shock loading over a long duration and is not intended to evaluate health effects from single or short-term shock events. However, the Standard gives a method to determine the spinal response as a function of the accelerations measured at the seat. This analysis observes the spinal response in the vertical direction, which is represented by a recurrent neural network given for data sampled at $50 \mathrm{~Hz}$ [7]. Data sampled at a higher frequency is down-sampled by first applying an anti-aliasing filter using a forward-backward second order low-pass Butterworth filter having an $80 \mathrm{~Hz}$ cut-off frequency. The data then are re-sampled to a new $160 \mathrm{~Hz}$ time vector using linear interpolation of the filtered data. Using the formulation in ISO 2631-5, the lumbar acceleration timehistory is determined, and the maximum value corresponding to lumbar compression is given as the output. For this study, the vertical lumbar acceleration is assumed proportional to the level of discomfort.

\subsubsection{Bandwidth-Limited PSD}

The BL PSD method was introduced by Peterson and Bass at the Naval Surface Warfare Centre - Panama City for application in the context of high speed craft [8]. The BL PSD is calculated as the average PSD value in the $4 \mathrm{~Hz}$ to $8 \mathrm{~Hz}$ band using a 4096-point Hamming window with 50\% overlap. This method can be modified to use a more appropriate frequency range and window function. A force window is the preferred window for a response to an impact [16] and is more appropriate for the case when traversing a speed hump. Further, the average PSD value in the range of $4 \mathrm{~Hz}$ to $10 \mathrm{~Hz}$ is taken, as the biodynamic response is not insignificant in the $8 \mathrm{~Hz}$ to $10 \mathrm{~Hz}$ range and must be considered [4], [12], [17].

\subsubsection{Acceleration Onset: Average Jerk}

The average jerk provides a measurement of the acceleration-onset. The rise-time, $t_{R}$, and peak acceleration are determined using the method of MIL-DTL-9479E [12]. The average onset jerk, $J_{\text {avg }}$, is defined as

$$
J_{\text {avg }}=\frac{a_{\text {peak }}}{t_{R}}
$$

Comfort and injury risk limits on this value derived from human and animal experiment data are reported in [13]. 


\section{Methods}

\subsection{Speed Hump Locations}

The experiments were conducted on a $2.7 \mathrm{~km}$ long circuit in a Type III ambulance that included three standard speed humps (labelled 1, 2, and 3) in the order in which they were encountered, and three flat-top speed humps (labelled A, B, and C) ordered similarly. The standard speed humps were located along Kingsdale Avenue between Bank Street and Conroy Road in Ottawa, Ontario, Canada, and the flat-top speed humps were located along Queensdale Avenue between Bank Street and Conroy Road. Speed hump locations and the route followed during the experiments are shown in Fig. 2.

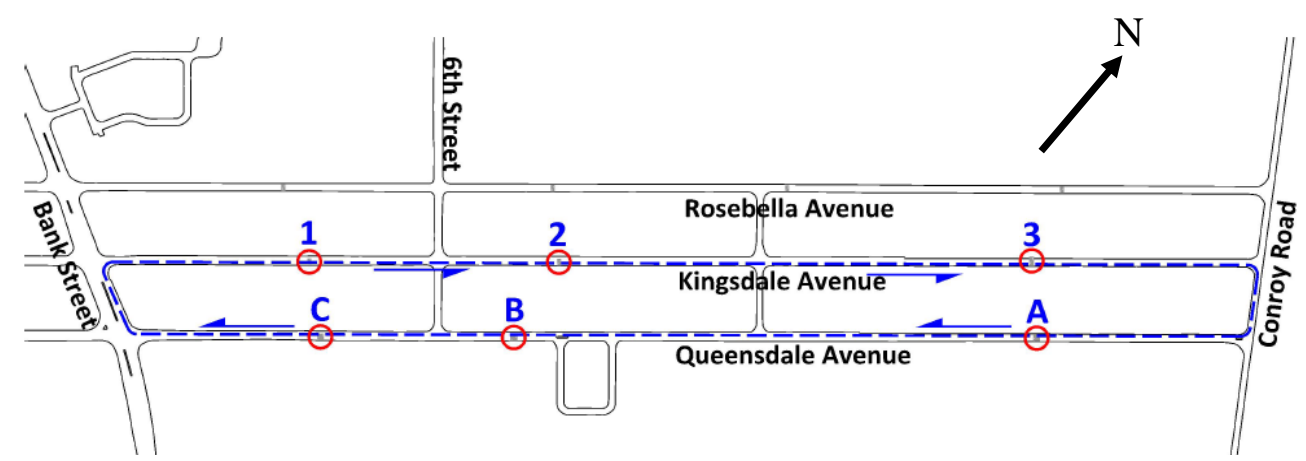

Fig. 2: Speed hump locations and the routes followed during the experiments. Retrieved from [18].

\subsection{Speed Hump Profile}

The City of Ottawa standard for a standard speed hump is a $4 \mathrm{~m}$ long hump with a sinusoidal profile [18]. A flat-top speed hump has a top section $3 \mathrm{~m}$ long between the two halves of a standard speed hump. All the hump profiles are $80 \mathrm{~mm}$ high. Due to construction tolerances, the actual completed profiles of the tested humps varied moderately, but within a reasonable range. The profiles of the speed humps tested are shown in Fig. 3, along with the standard profile. The heights of standard speed humps 1 and 3 are higher than the city standard, at $88 \mathrm{~mm}$ and $90 \mathrm{~mm}$, respectively. The height of standard speed hump 2, at $79 \mathrm{~mm}$, is very close to the standard. The overall shapes of the standard speed humps were close to roundtop rather than sinusoidal profiles.

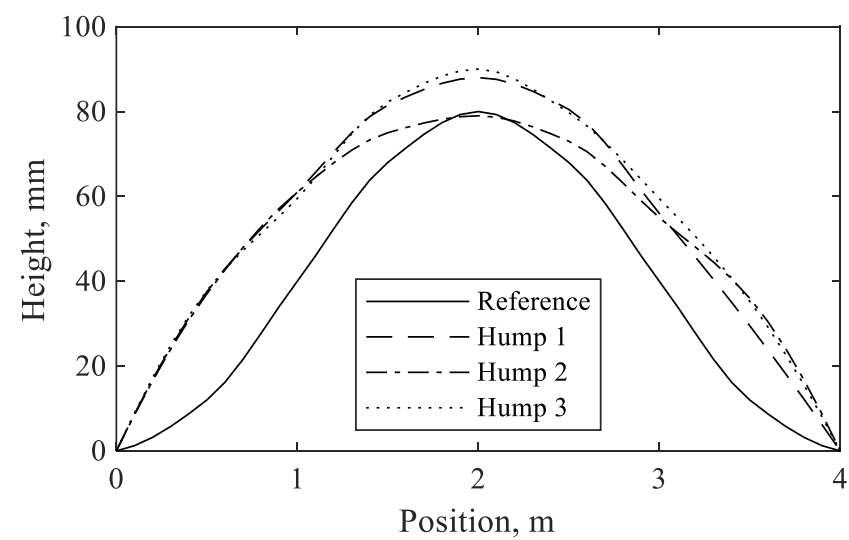

a) Sinusoidal profile

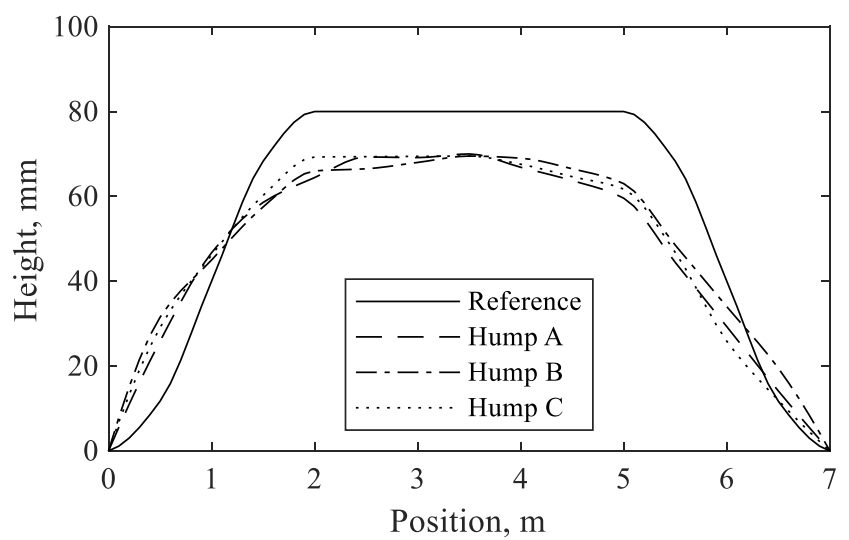

b) Flat-top profile

Fig. 3: Speed hump profiles.

\subsection{Instrumentation}

The ambulance was equipped with a LORD Microstrain 3DMGX3 inertial measurement unit (IMU), which was GPS enabled, and was mounted directly on the vehicle's floor near the vehicle's centre of mass. 


\section{Results}

On-road data collection was completed in Ottawa, Ontario, Canada on 27 November 2018 between 12:00PM and 1:00PM. Road conditions were typical, with some light snow cover and no significant traffic. The ambulance driver was instructed to traverse the speed humps at 6 different speeds: 15, 20, 25, 30, 35, and $40 \mathrm{~km} / \mathrm{hr}$. The $30 \mathrm{~km} / \mathrm{hr}$ loop was conducted twice since this corresponded with the advisory speed for the speed humps.

Acceleration along the z-axis (vertical) recorded from the ambulance floor is shown in Fig. 4 with the speed hump data highlighted in red and blue. The acceleration data from the IMU was recorded at approximately $100 \mathrm{~Hz}$ with slight variations in sampling rate. The data were therefore interpolated to a constant sampling rate of $100 \mathrm{~Hz}$. Acceleration in the z-axis was also offset by the gravitational constant. The centre timestamp of each speed hump was determined by taking the average timestamp of GPS coordinates within $6 \mathrm{~m}$ of the speed hump coordinate. The speed hump segment is defined as 1 second before and 2 seconds after the centre timestamp. The speed during each segment was calculated by taking the average speed within the segment as reported by the GPS and integrated within the IMU sensor. For each of these segments, the five human comfort parameters were calculated. The sensor was placed directly on the ambulance floor and attenuation that may result between the ambulance floor and bench seat, on which a paramedic sits when attending to a patient, was neglected since it is rigidly attached to the ambulance floor with only thin padding on the top. The results from this analysis therefore provide a worst-case evaluation of these parameters.

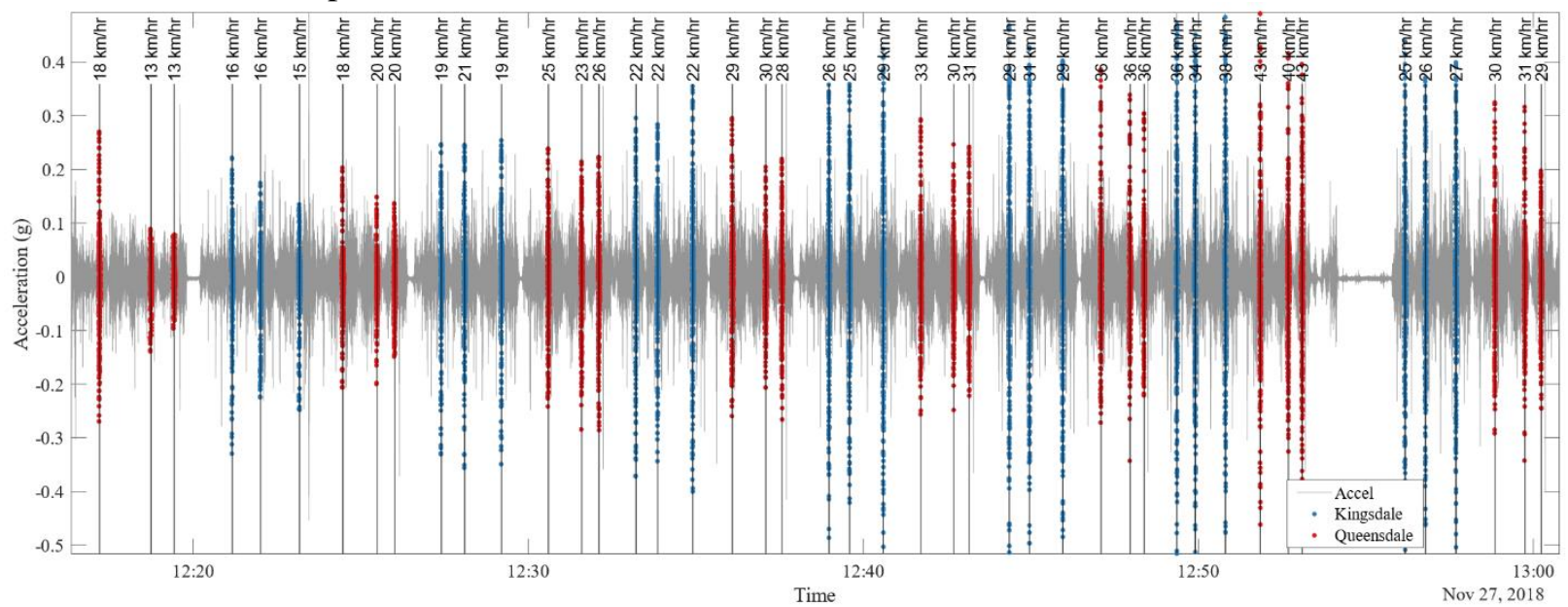

Fig. 4: Acceleration time-history measured at the ambulance floor in the vertical $z$-axis.

\subsection{Peak Seat Acceleration}

The peak seat acceleration for each of the speed hump traversing speeds is shown in

Fig. 5:. The average linear correlation coefficient between acceleration and speed for the sinusoidal speed humps is 0.96 and the average correlation for the flat-top speed humps is 0.95 . There is a strong positive linear relationship between the traversing speed and the peak acceleration. There is also low variability of this parameter across the different speed humps. Based on a study by Kjemtrup, trial participants responded that a vertical acceleration value over $0.2 \mathrm{~g}$ will cause some degree of discomfort, with more significant discomfort over $0.4 \mathrm{~g}$ [19]. According to this parameter threshold, traversing speed humps over $20 \mathrm{~km} / \mathrm{hr}$ will cause discomfort, with significant discomfort over $30 \mathrm{~km} / \mathrm{hr}$. Notably, flat-top speed humps caused slightly lower peak accelerations than sinusoidal humps overall.

\subsection{Dynamic Response Index}

The resulting DRI for each of the speed hump traverses at the varying speeds is shown in Fig. 6. The average linear correlation coefficient for the sinusoidal speed humps is 0.96 and the average correlation for the flat-top speed humps is 0.79 . There is a strong positive linear relationship between the traversing speed and the DRI. Across the sinusoidal speed humps there is less variation in DRI compared to across the flat-top humps. DRI is also lower overall for the flat-top speed humps. The DRI values from traversing the speed humps is well below the threshold for passenger comfort at all speeds, which is 
around 3 for low quantity exposure [10]. Even at high quantity exposures (i.e. 1000 shock events in 24 hours), the DRI from the speed humps does not exceed the comfort limit.

\subsection{Peak Lumbar Acceleration}

The peak lumbar acceleration for each of the traversing speeds is shown in Fig. 7. The average linear correlation coefficient for the sinusoidal speed humps is 0.95 and the average correlation for the flat-top speed humps is 0.81 . There is a strong positive linear relationship between the traversing speed and the peak lumbar acceleration. Across the sinusoidal speed humps there is less variation and a stronger trend compared to across the flat-top humps. The peak lumbar acceleration is also lower overall for the flat-top speed humps. The trends observed in the peak lumbar acceleration are similar to those observed in the DRI.

\subsection{Bandwidth-Limited PSD}

The BL PSD for each of the traversing speeds is given in Fig. 8. The average linear correlation coefficient for the sinusoidal speed humps is 0.81 and the average correlation for the flat-top speed humps is 0.88 . There is high variation between individual humps for both types of speed humps. There is a positive linear relationship between the traversing speed and the BL PSD, but the strength of this relationship varies across the individual speed humps. BL PSD is in general, lower for flat-top speed humps.

\subsection{Average Jerk}

The average acceleration onset jerk for each of the traversing speeds is shown in Fig. 9. The average linear correlation coefficient for the sinusoidal speed humps is 0.02 and the average correlation for the flat-top speed humps is -0.51 . The relationship between average jerk and traversing speed is weak for both types of speed humps. The relationship appears to be negative due to a few high-valued points at low speeds. This could be an indication of increased discomfort at low speeds.

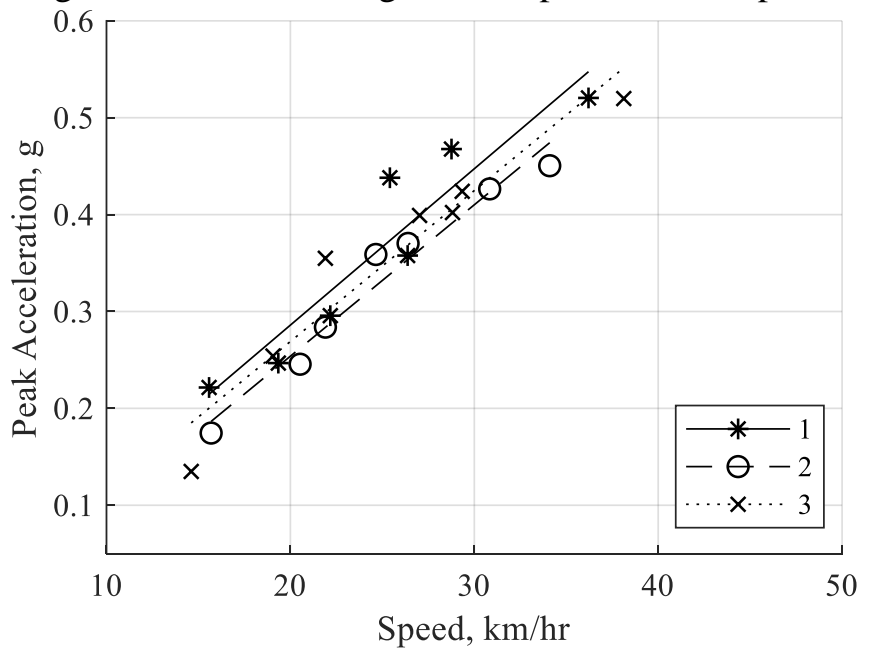

a) Sinusoidal profile

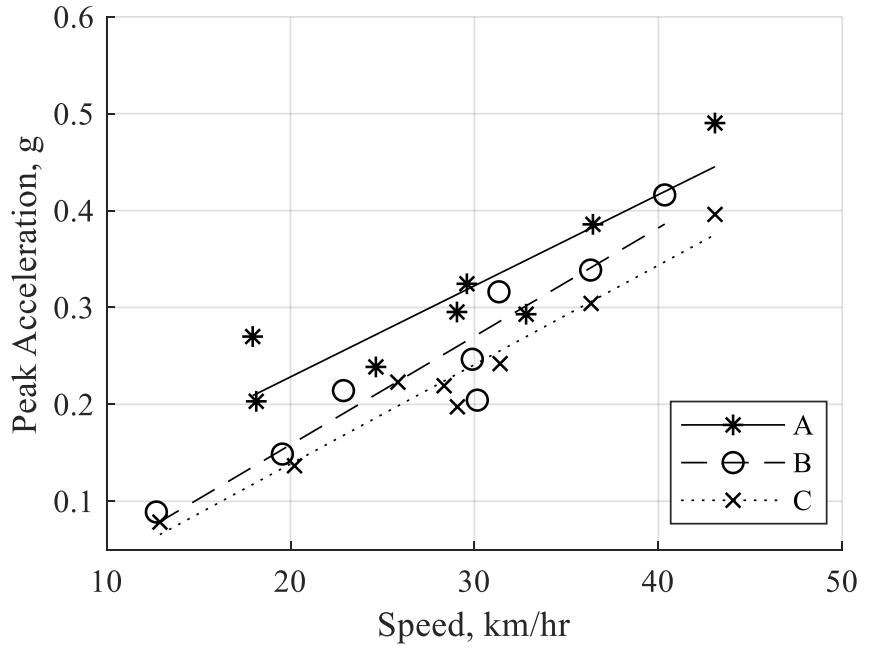

b) Flat-top profile

Fig. 5: Peak acceleration measured at the ambulance floor. 


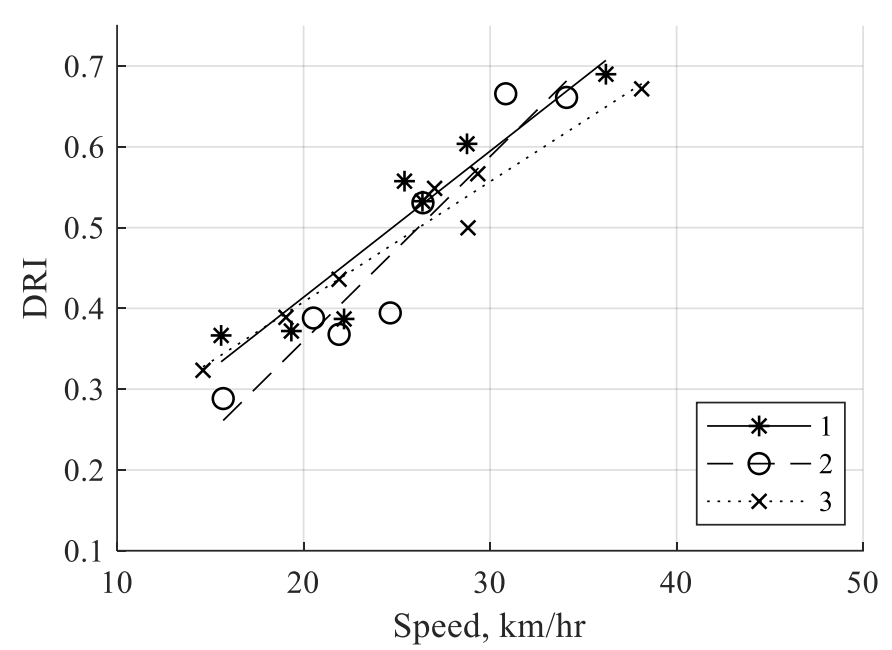

a) Sinusoidal profile

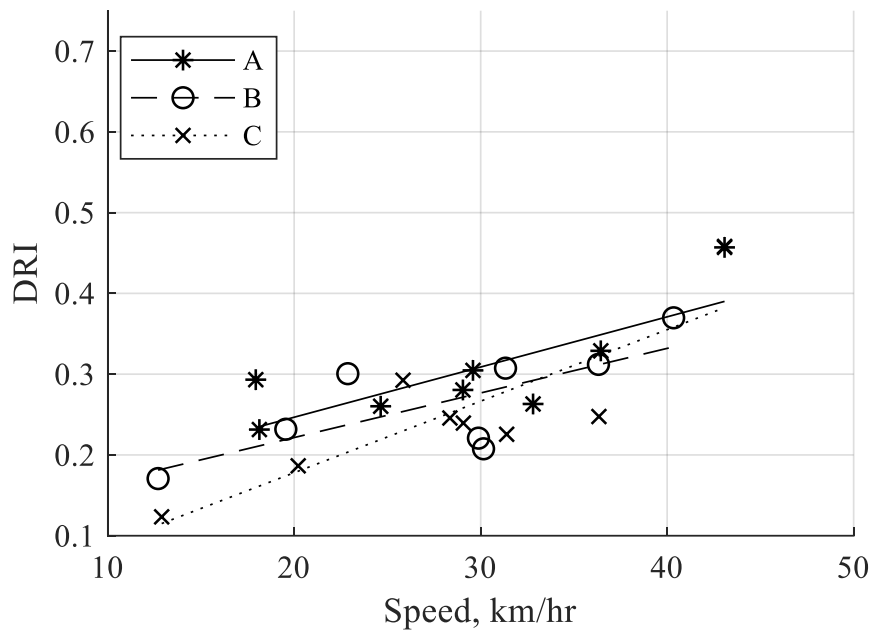

b) Flat-top profile

Fig. 6: DRI measured at the ambulance floor.

\section{Discussion and Conclusion}

All parameters provided unique insight to the speed hump traversal event and showed a strong positive linear correlation with the traversal speed apart from the average jerk. Average jerk resulted in some elevated values at low speeds that may reflect limitations of the suspension system to absorb low-speed heave-dominated shock events. The DRI and peak lumbar acceleration are highly correlated and provide similar information. The peak acceleration showed the strongest relationship with the speed of traversal when compared with the other parameters and seems to be the most robust parameter against noise. This is likely due to the fact that the evaluation of peak acceleration is not dependent 


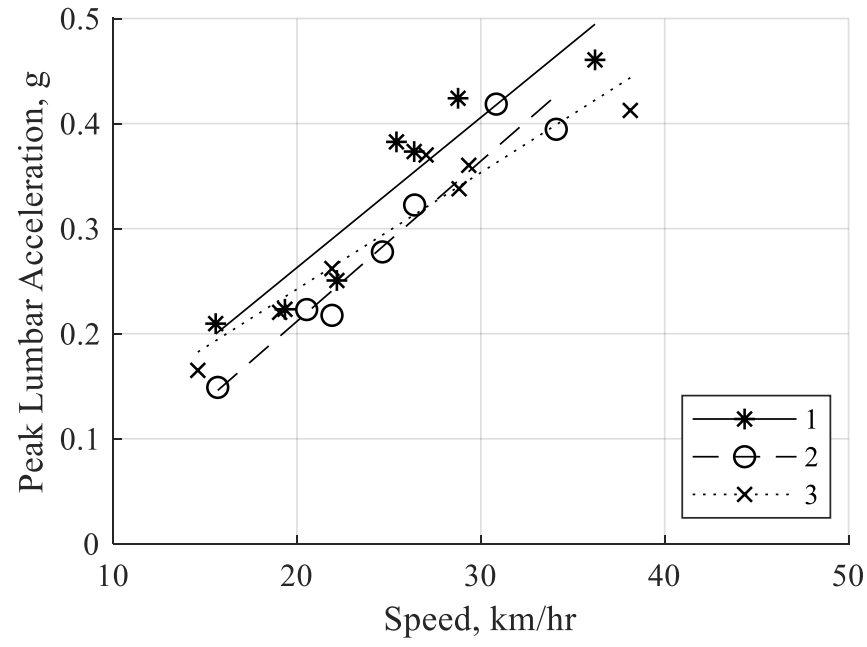

a) Sinusoidal profile

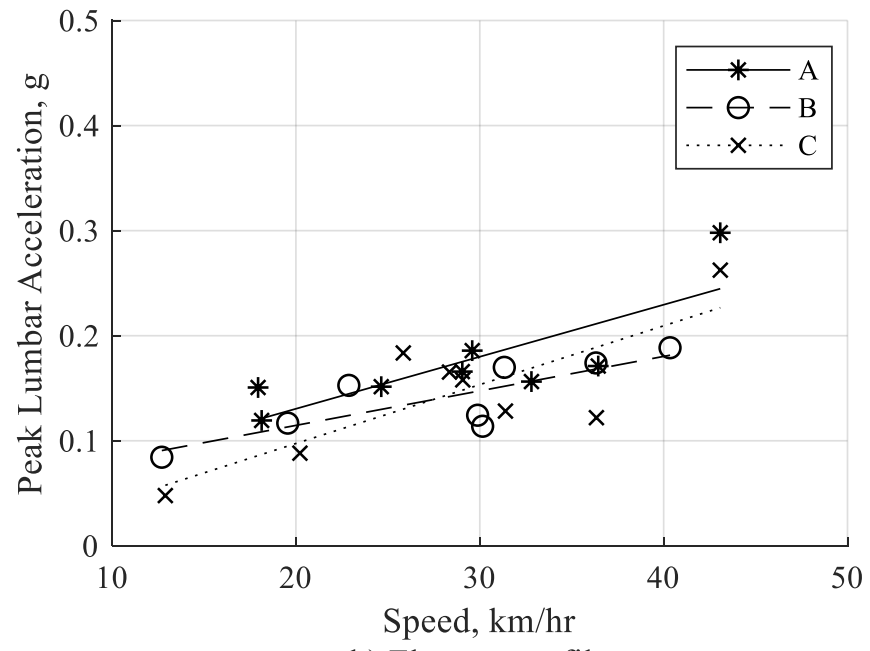

b) Flat-top profile

Fig. 7: Peak lumbar acceleration (from ISO 2631-5) measured at the ambulance floor.

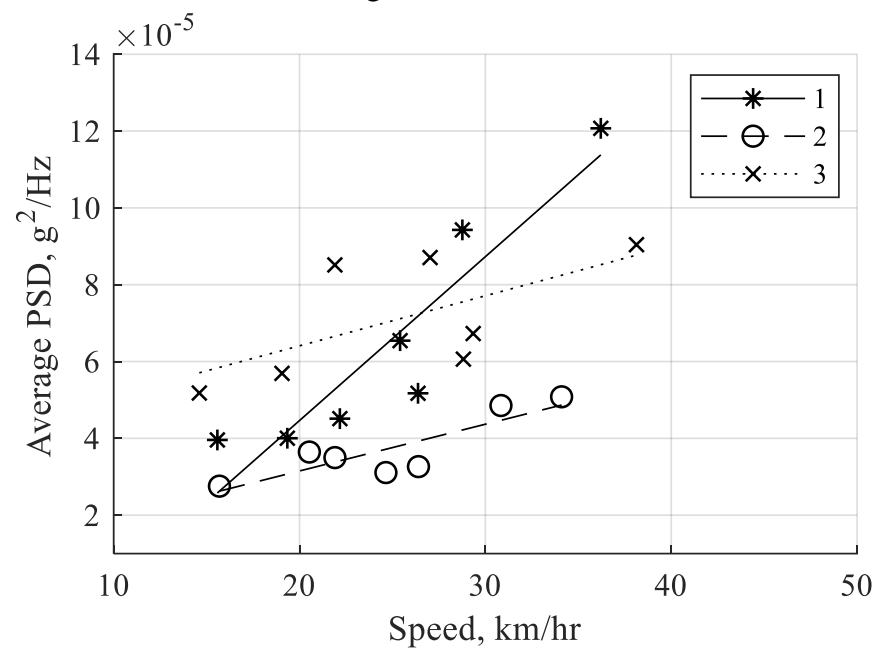

a) Sinusoidal profile

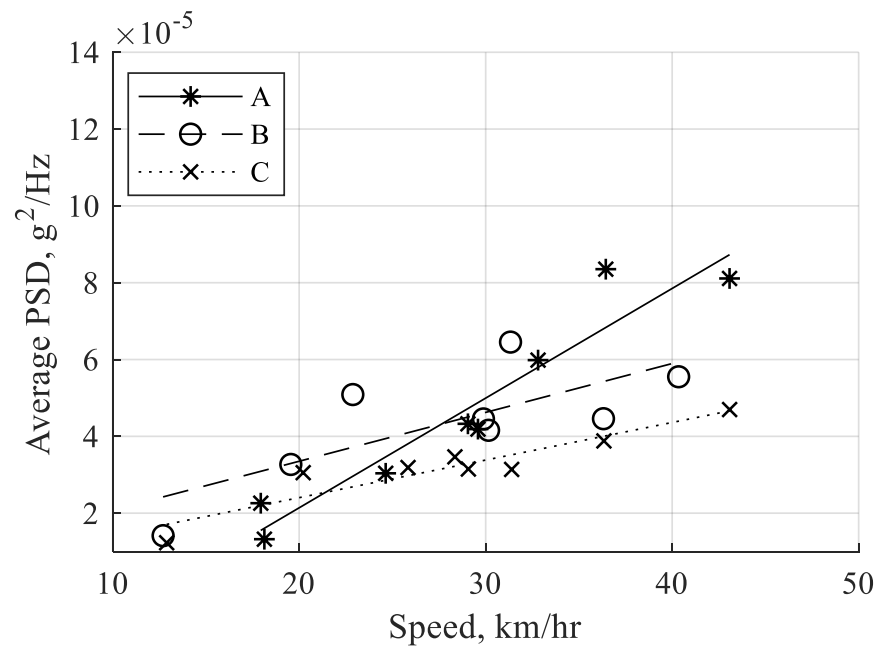

b) Flat-top profile

Fig. 8: Average BL PSD value measured at the ambulance floor. 


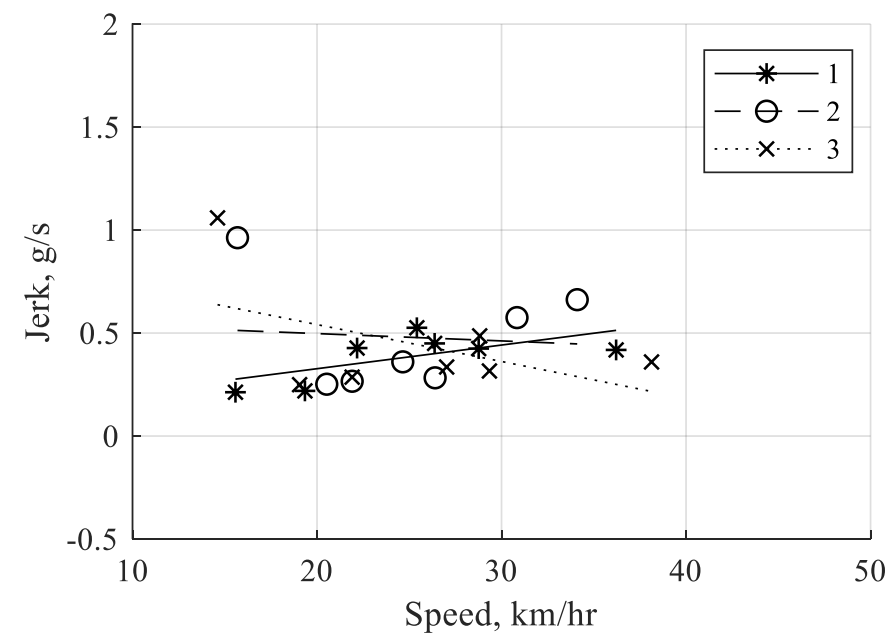

a) Sinusoidal profile

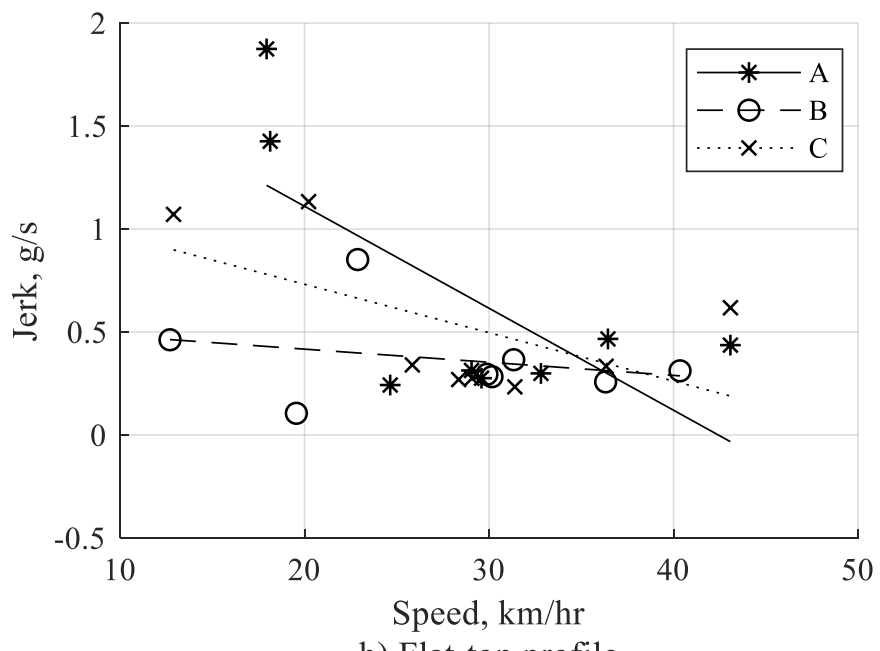

b) Flat-top profile

Fig. 9: Average acceleration onset jerk measured at the ambulance floor.

On the length of the time series used, as long as the peak occurs within that segment. Other parameters are evaluated using the entirety of the time series and therefore should be more sensitive. The increased sensitivity from these parameters can provide additional value. For the DRI and peak lumbar acceleration, the difference between the two types of speed humps is more evident than in the peak acceleration. As shown in Fig. 3, each speed hump has a unique profile and this difference is not captured in the peak acceleration. On the other hand, the BL PSD shows an evident separation between each individual speed hump.

Several of the parameters considered in this study were derived and applied in military settings and other extreme environments to assess the injury risk. The novel application of these parameters to ambulance transport and the speed hump traversal event provided additional insight to the human biodynamic response and showed that a single parameter in isolation, such as the peak acceleration, is insufficient to adequately assess comfort. Future work would involve running multiple trials of these traversals in order to better evaluate the consistency of these parameters. Additional sensors attached to other points on the ambulance (e.g. passenger seats) would be included, to provide a better understanding of the accelerations experienced throughout the ambulance.

\section{Acknowledgements}

The authors would like to acknowledge the City of Ottawa, the Ottawa Paramedic Service, the Children's Hospital of Eastern Ontario, Roger Selzler, and Fadwa Darwaish for their participation in the data collection.

\section{References}

[1] D. Zaidel, A. S. Hakkert, and A. H. Pistiner, "The use of road humps for moderating speeds on Urban streets," Accid. Anal. Prev., vol. 24, no. 1, pp. 45-56, Feb. 1992, doi: 10.1016/0001-4575(92)90071-P.

[2] M. Martens, "The Effects of Road Design on Speed Behaviour: A Literature Review," 1997.

[3] M. Belchamber, "A study of paramedics' attitudes to the effects of speed humps on resuscitation of patients en route to hospital, including general patients care and ambulance response times," May 2003.

[4] International Organization for Standardization, ISO-2631-1, Mechanical vibration and shock - Evaluation of human exposure to whole-body vibration. Geneva, 1997.

[5] V. Jasiūnienè, G. Pociūtè, A. Vaitkus, K. Ratkevičiūte, and A. Pakalnis, "Analysis and evaluation of trapezoidal speed humps and their impact on the driver," Balt. J. Road Bridg. Eng., vol. 13, no. 2, pp. 104-109, 2018, doi: 10.7250/bjrbe.2018-13.404.

[6] A. Gedik, E. Bilgin, A. H. Lav, and R. Artan, "An investigation into the effect of parabolic speed hump profiles on ride comfort and driving safety under variable vehicle speeds: A campus experience," Sustain. Cities Soc., vol. 45, pp. 413- 
421, Feb. 2019, doi: 10.1016/j.scs.2018.11.040.

[7] International Organization for Standardization, ISO-2631-5, Mechanical vibration and shock - Method for evaluation of vibration containing multiple shocks. Geneva, 2004.

[8] R. Peterson, E. C. Pierce, B. Price, and C. R. 'Dale' Bass, "Shock Mitigation for the Human on High Speed Craft: Development of an Impact Injury Design Rule," 2004.

[9] P. De Alwis, "Methods for Shock and Vibration Evaluation Applied on Offshore Power Boats," 2014.

[10] H. E. von Gierke and A. J. Brammer, "Effects of Shock and Vibration on Humans," in Harris' Shock and Vibration Handbook, 6th ed., McGraw-Hill Education, 2009.

[11] J. Li, W. Guo, L. Wang, and S. Chen, "Multi-objective optimization of ambulance ride comfort under speed bump," IEEJ Trans. Electr. Electron. Eng., vol. 14, no. 9, pp. 1372-1380, Sep. 2019, doi: 10.1002/tee.22939.

[12] "MIL-DTL-9479E: DETAIL SPECIFICATION: SEAT SYSTEM, UPWARD EJECTION, AIRCRAFT, GENERAL SPECIFICATION FOR," 2007.

[13] A. M. Eiband, "Human Tolerance to Rapidly Applied Accelerations: A Summary of the Literature," 1959.

[14] NATO Standardization Agency, "Allied Engineering Publication AEP-55 (C), Volume 3 on 'PROCEDURES FOR EVALUATING THE PROTECTION LEVEL OF ARMOURED VEHICLES."”

[15] G. Allen, "The Use of a Spinal Analogue to Compare Human Tolerance to Repeated Shocks with Tolerance to Vibration," in AGARD-CP-253: Models and Analogues for the Evaluation of Human Biodynamic Response, Performance and Protection, Neuilly Sur Seine, France: North Atlantic Treaty Organization, 1978.

[16] W. A. Fladung, "Windows used for impact testing," SPIE Int. Soc. Opt. Eng., pp. 1662--1666, 1997.

[17] W. Abbas, O. B. Abouelatta, M. El-Azab, M. Elsaidy, and A. A. Megahed, "Optimization of Biodynamic Seated Human Models Using Genetic Algorithms," Engineering, vol. 02, no. 09, pp. 710-719, 2010, doi: 10.4236/eng.2010.29092.

[18] W. Langlois, Robert G., Kang, "Measurement of Ride Quality Data in Support of the City of Ottawa's 'Impacts of a Speed Hump on Transit Vehicles Study,", Ottawa, Ontario, ADL/2017/RL/1, 2017.

[19] K. Kjemtrup, "Speed reducing measures," in Australian Road Research Board (ARRB) Conference, 14th, 1988, pp. $125-135$. 